\title{
Outlaw Community Innovations
}

\author{
Celine Schulz and Stefan Wagner
}

Discussion paper 2008-08

July 2008

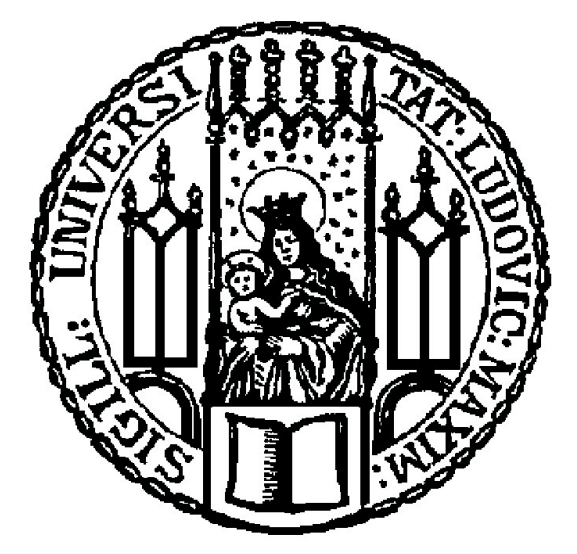

Munich School of Management

University of Munich

Fakultät für Betriebswirtschaft

Ludwig-Maximilians-Universität München

Online at http://epub.ub.uni-muenchen.de/ 


\title{
Outlaw Community Innovations*
}

\author{
Celine Schulz and Stefan Wagner ${ }^{\dagger}$ \\ University of Munich
}

1, July 2008

\begin{abstract}
Recent studies of outlaw communities provide qualitative evidence of their existence and the organisation of the underlying innovation processes. We provide descriptive results from a large scale survey of two online outlaw communities focussing on Microsoft's XBox. In line with previous findings, we identify two types of participants in outlaw communities - user innovators and adopters. Based on 2,256 responses, we find that users modify their XBox mainly to be able to increase the set of available functions of their XBox. Users are also motivated to modify their XBox for the sake of having fun and to conduct pirate behaviour. Finally, the results from our survey suggest that user innovators are largely intrinsically motivated by fun and the intellectual stimulation of writing code for homebrew software.
\end{abstract}

Keywords: Outlaw community innovation, video game consoles, homebrew software

*We greatly acknowledge helpful discussions with Dietmar Harhoff and Ulrich Lossen. Further we would also like to thank our colleagues at INNO-tec and participants of the TIME seminar at the TU Munich and participants of the 'Conference on IP and the creative industries' in London 2008.

$\dagger$ Institute for Innovation Research, Technology Management and Entrepreneurship, Munich School of Management, Kaulbachstraße 45, D-80539, Munich, Tel: +49 892180 3760, e-mail: cschulz@bwl.uni-muenchen.de 


\section{Introduction}

User innovation is a commonly observed phenomenon in various industries where users modify or improve products that they use (von Hippel 1988, 2005). Communities of user innovators - innovation communities - provide platforms for users to openly and voluntarily communicate with each other regarding innovations they are working on either collectively or independently (Franke \& Shah 2003, Hienerth 2006, von Hippel 2005). Recent studies that have examined the relationship between innovation communities and firms have found that there is often a symbiotic relationship from which both users and manufacturers benefit (Jeppesen \& Molin|2003, Jeppesen \& Frederiksen|2006, Prügl \& Schreier 2006). Dahlander \& Magnusson (2005) distinguish between commensalistic (where the manufacturer gains and the community is indifferent) and parasitic (where the manufacturer gains on the expense of the community) relationships.

However, there could be an additional type of relationship where community innovations can be beneficial for users and at the same time harmful for manufacturers. An example of such a relationship is when innovations stemming from communities aim at bypassing legal or technical safeguards that prevent users from unsolicited usage of the manufacturer's products (Mollick 2004). In particular, manufacturers of consumer electronic devices often install security mechanisms to prevent users from executing unauthorised software code or DRM-protected content on their platform (such as pirated copies of authorized software or illegal copies of MP3s). Research on user innovations that deactivate such security mechanisms in order to give the user full control over the usage of the product was first introduced by Mollick (2004). Extending this research, Flowers (2006) coined the term outlaw innovation and provided case studies of how communities create and disseminate innovations that not only conflict with manufacturers' intensions of the usage of the original product but also violate firms' intellectual property rights. He proposes that outlaw communities consist of both users who innovate and those who simply adopt and use outlaw innovations. Recent examples of such outlaw communities in the consumer electronics industry include www.XBox-scene.com, www.XBox-linux.org and www.free60.org for the Microsoft XBox (a gaming console); www.iphonehacks.com for Apple's iPhone (a mobile phone); and www.cellphonehacks.com for cell phones in general. In the examples listed above, user innovators were able to 'hack' (disable) security mechanisms enabling them (and other users) to run both user written software code (homebrew) and pirated programs on the now unprotected hardware.

Although both Mollick (2004) and Flowers (2008) perceive outlaw innovation as potentially harmful for the manufacturer, both propose strategies as to how firms can respond to these outlaw innovations in such a way that they can be profitable for their own innovation processes. But before firms can leverage outlaw communities for innovation management, they need to have a better comprehension of the phenomenon 
of outlaw innovation so that they can relate more effectively to it. It is thus important to firstly understand more about the actual individuals who modify and hack their products. To address these issues, our study aims to investigate the characteristics of the outlaw innovations implemented by users and the motivations that drive users to develop and adopt outlaw innovations.

In our paper, we provide results from an online-survey of users of two outlaw communities focusing on Microsoft's XBox. In total, we received 2,256 questionnaires from our online-survey posted at www.XBox-scene.com and www.XBox-linux.org. About $20 \%$ of the responses originated from users who actively contributed to modifications of original software or to self-written (so called homebrew) software. Results indicate that users modify their XBox mainly to be able to increase the set of available functions of their XBox. Next, users are motivated to modify their XBox for the sake of having fun and to be able to run pirated games. We also find that user innovators are largely intrinsically motivated by fun and the intellectual stimulation of writing code for homebrew software.

The remainder of this paper proceeds as follows: in section 2 we provide an overview of relevant literature on user communities and innovation; section 3 introduces the object of study - Microsoft's XBox and online-based outlaw communities focussing on the XBox's modification; the results of our survey are presented in section 4; section 5 concludes with a summary of the major findings and provides a short outlook on future research topics.

\section{Outlaw Communities}

User communities can be defined as horizontal user networks that consist of user nodes interconnected by information transfer links which may involve face-to-face, electronic or any other form of communication that provide members sociability, support, a sense of belonging, and social identity (Wellman et al. 2002, von Hippel 2007). Three conditions are necessary for user communities to function entirely independently of manufacturers: 1) some users innovate, 2) some users freely reveal ${ }^{1}$ their innovations, and 3) users can self-manufacture their innovations relatively cheaply (von Hippel 2007).

Communities can either focus on a common interest or on one or more products of a particular manufacturer. The first refers to special interest communities, such as sports communities where members interact with each other to exchange information and to assist each other with the development of their sports-related consumer product innovations (Franke \& Shah 2003, Shah 2005a, Hienerth 2006). The second type of communities consist of individuals who all use a similar product and who learn how to use it better as they interact with others on a regular basis (Wenger 2004). Examples

\footnotetext{
1"Free revealing" is defined as the granting of access to all interested agents without imposing any direct payments.
} 
of such user communities that have been examined in the literature include a firmestablished user community for computer-controlled music instruments (Jeppesen \& Frederiksen 2006), user communities in the video gaming industry (Prügl \& Schreier 2006), a statistical software community (Mayrhofer 2005) and user communities of a proprietary software firm (Schulz 2006).

In the above-mentioned cases for communities that focus on products of a particular manufacturer, it was found that both the participants of the communities as well as the manufacturers of the underlying product profit from the existence of organised innovation communities. In particular, participants not only benefit from the userto-user support but also from the gain in reputation they receive from the firm and other community members. When user innovators in these communities freely reveal their innovations, firms profit by implementing these innovations into their proprietary products and selling them to all users. (Mayrhofer 2005, Jeppesen \& Frederiksen 2006)

However, user communities may not always be beneficial for the manufacturer of the underlying product per se. Our study focuses on communities that exist independently from the manufacturer and whose members create innovations that aim at bypassing legal or technical safeguards that prevent users from unsolicited usage of the manufacturer's product (Mollick 2004). Specifically, we examine one particular form of user community innovation - hacking, which refers to user modifications of a product to not only gain unauthorised access to the product's system but to also enable the user to use the system more effectively (Meyer 1989). According to Mollick (2004), hackers can be categorised into innovative "Elites" who understand the proprietary system and cause it to do things its makers never intended, and follower "Kiddies" who do not truly understand the system, but merely use tools created by the Elites in order to exploit the system in their own way. Flowers (2008) generalises these concepts and defines outlaw communities as consisting of both users who are user innovators (Elites) and adopters of these user innovations (Kiddies). Specifically, outlaw communities can be defined as groups of users who create and disseminate innovations that not only conflict with manufacturers' intentions of the usage of the original product but also violate firms' intellectual property rights.

Software hackers initiated the open source movement in the late 1990s to promote open collaborative projects between software developers (Raymond 1999). Although open source software communities today are widely perceived to be legitimate and "aboveground" (Mollick 2004), it is nevertheless useful to examine the motivations that drive software developers (user innovators) to contribute to open source software projects as they may be relevant for analysing outlaw communities. Theoretical and empirical studies show that these incentives for contribution can be categorised into intrinsic and extrinsic motivations. Extrinsic motivation refers to the separable outcome (or indirect reward) that is attained when an activity is done, whereas intrinsic motivation is the inherent satisfaction of the doing of an activity (Ryan \& Deci 2000). 
Examples of extrinsic motivations include skill improvement through active peer review (Hars \& Ou 2001, Ghosh et al. 2002) and the signaling motive for career advancements and/or future career benefits (Lerner \& Tirole 2001, 2002). Creativity (Lakhani \& Wolf 2005), fun (Torvalds \& Diamond 2001, Lakhani \& Wolf 2005, Bitzer et al. 2004), reputation (Raymond 1999, Ghosh et al. 2002, Lakhani \& Wolf 2005), altruism (Zeitlyn 2003, Bitzer et al. 2004) and reciprocity (Lakhani \& von Hippel 2003) are examples of intrinsic motivations.

In his study of four outlaw communities, Mollick (2004) finds that user innovators are intrinsically motivated by the desire to discover and innovate, rather than for the sake of theft. Adopters on the other hand are more often "vandals" who are motivated to adopt an outlaw innovation to gain unauthorised access to their product's system for pirate behavior. Despite these differences, the user innovators are willing to allow the adopters to use their innovation so as to gain attention to their work and to give them a certain sense of satisfaction, i.e. they benefit from the gain in reputation. This symbiotic relationship between the innovators and the adopters promotes the diffusion of outlaw innovations.

Differentiating between user innovators and adopters, we aim to investigate the characteristics of outlaw innovations which are implemented by community members and the motivations that drive individuals to develop and adopt outlaw innovations. In particular, we hope to be able to provide a deeper understanding regarding the inherent nature of the outlaw innovation, that is if it is intrinsically "harmful" or if they are just a result of creative individuals having fun.

\section{Survey Design}

\subsection{Object of study - the case of Microsoft's XBox}

The phenomenon of outlaw community innovation has already been identified and described in the literature (Mollick 2004, Flowers 2008). However, until now it remains largely unclear what exactly drives individuals to participate in such communities and to what extent the two different types of community participants (user innovators and adopters) described by Mollick (2004) and Flowers (2008) do coexist in outlaw communities. While survey evidence of innovation communities exists (Franke \& Shah 2003, Shah 2005a, Hienerth 2006, Jeppesen \& Frederiksen 2006, Prügl \& Schreier 2006) we present results from the first survey of participants of two large internet-based outlaw innovation communities focused on Microsoft's XBox. Before advancing to the description of the survey design applied, we would like to provide some background information on the emergence of user modifications of various electronic products in general and in particular, modifications of the XBox.

Firstly, it is important to note that the technical architecture of many consumer 
electronic products is based on the design of standard personal computers. They contain key building blocks like a Central Processing Unit (CPU) which processes commands initiated by an (often embedded) operating system ${ }^{2}$, memory devices for manipulating and storing data and different types of input/output devices such as displays and controllers or keyboards. In general, the embedded operating system (often called firmware) allows users to execute a closed set of exactly specified functions on the hardware. ${ }^{3}$ The set of defined functions provided by the firmware is only a subset of all functions which could in theory be performed by an electronic device. Sometimes, consumer electronic devices like video gaming consoles or mobile phones also allow the execution of third-party applications like games or other pieces of software. However, the execution of additional applications is often restricted by security mechanisms requiring third-party developers to acquire costly licenses from the device's manufacturer to ensure compatibility with the embedded firmware.

Most user innovations occur in this context as attempts to manipulate or to replace the embedded operating system in order to extend the limited set of functions provided by the manufacturer with additional functions or to disable security mechanisms to allow for the execution of unauthorised pieces of software as well as illegal copies of authorised software. The manipulations of the firmware which bypasses security mechanisms largely resembles the hacking of computer systems and is therefore often regarded as an outlaw activity (see Flowers (2008) for a more detailed discussion). Known examples of products where users provided modifications/ hacks of the embedded operating system range from mobile phones (Apple's iPhone has been hacked in order to allow for the execution of not authorised third party applications ${ }^{4}$, to hard-disk based VCRs ${ }^{5}$ and also network controllers ${ }^{6}$.

It should be noted though that video gaming consoles and video games themselves have historically been a target of user modifications since their emergence (see Levy (1984) for a detailed overview). In general, consoles are a particularly attractive target for user modifications for several reasons: First, gaming consoles are in general powerful computing devices which are often equipped with high performance CPUs and graphic processors in order to cope with computational intense high-definition graphics of video games. As a consequence, gaming consoles can in principle execute a wide range of different applications without running into computational restrictions. Sec-

\footnotetext{
${ }^{2}$ An operating system can be defined as embedded if it is contained inseparably in a device. Often embedded operating systems are stored on ROM or Flash memory chips and can not easily be replaced by the user.

${ }^{3}$ In the following, "firmware" specifies the operating system embedded in an electronic device by its manufacturer.

${ }^{4}$ See http://www.iphonehacks.com for more details. Latest visit on March $26^{\text {th }} 2008$.

${ }^{5}$ See http://www.lugod.org/presentations/tivohacks for more details. Latest visit on March $26^{\text {th }} 2008$.

${ }^{6}$ See http://www.nslu2-linux.org/wiki/Unslung/HomePage for more details. Latest visit on March $26^{\text {th }} 2008$.
} 
ond, gaming consoles are often sold at a subsidised price as manufacturers anticipate significant revenue streams from complementary products such as software, games or controllers (Soghoian 2007). In many cases, purchases of hardware with similar technical specifications would be possible only at higher prices.

While user modifications exist for almost all major video gaming consoles Grand et al. 2004), we will focus on Microsoft's XBox for a number of reasons. ${ }^{7}$ When it was released in 2001, the XBox was equipped with a $733-\mathrm{MHz}$ Intel Celeron processor, a nVidia GeForce GMX Graphics Processing Unit running at $233 \mathrm{MHz}$, a 100 MBit/ second ethernet interface and a 10 GB hard disk (see Grand et al. (2004) for a detailed description of the technical specifications of the XBox). This configuration easily topped even upper-scale desktop computers sold at the same time. ${ }^{8}$ Despite offering immense computational power, the initial recommended price of an XBox was relatively low with USD 299,- in the US as compared to personal computers with a similar performance. ${ }^{9}$ Due to the PC-like architecture and its comparably low price, the XBox quickly drew the attention of user innovators trying to disable Microsoft's security mechanism which was eventually hacked at the end of 2001 (Takahashi 2002). As a consequence, users where able to execute non-authorised software (including illegal copies of authorised software) on the XBox given that they applied appropriate hacks to disable Microsoft's security mechanism. ${ }^{10}$

After the XBox's security mechanism was hacked, numerous pieces of software provided by individual users emerged (so called homebrew software). As it would be far beyond the scope of this paper to provide a detailed overview on the XBox homebrewscene as a whole, we restrict ourselves to the organisation of developers and adopters of homebrew software in internet-based communities where information on the software is discussed and distributed. The development of homebrew software within these communities often follows the principles of open source software development where a larger group of developers collaborate on a larger software suites which source code is put into the public domain. One of the most successful homebrew applications for Microsoft's XBox is the XBox Media Center (XBMC) (see www . xbmc . org for a detailed description) - a very comprehensive multi-media application providing possibilities to store, administrate and play different types of multi-media files including audio and video files. Note that the original XBox firmware provided by Microsoft provides only very limited possibilities to exploit the multi-media skills of the XBox's hardware. It offers the possibility to play and store music from CDs inserted directly to the

\footnotetext{
${ }^{7}$ We focus on the first-generation XBox. The current version of the XBox ('XBox 360') was introduced in December 2005.

${ }^{8}$ Due to the comparably long life cycles of gaming consoles - for example, the XBox was sold until 2005 with an unchanged hardware configuration - manufacturers include state-of-the-art technology when launching new products.

${ }^{9}$ See http://en.wikipedia.org/wiki/XBox, latest visit February, $26^{\text {th }}, 2008$.

${ }^{10}$ The XBox's security mechanism can be disabled by overwriting an internal flash memory chip, by adding an additional chip or by applying a software-based hack.
} 
XBox with limited functionality with regard to manage larger collections of songs. The playback of DVDs is only possible if an additional controller has been purchased by the user. The XBMC extends this limited set of multi-media skills of the XBox with various functions and can therefore be seen as users' efforts to increase the set of available functions. It is worth emphasising that the collaborative effort in this project yielded a multi-media package whose quality is above the standard of comparable players provided for PC operating systems - in fact, the XBMC is so successful that it has even been ported to the Apple OS.

\subsection{Survey Design}

To date, studies on the phenomenon of outlaw innovation focused on qualitative case studies (Mollick 2004, Flowers 2008) and there is little systematic large scale evidence with respect to characteristics of users engaged in such activities or their motivations. In order to shed some more light into these questions we conducted an online survey of internet communities focussing on homebrew software for the XBox. We surveyed participants of two relevant online communities using an anonymous online questionnaire hosted on servers of the University of Munich between March, $15^{\text {th }}$ and April $3^{\text {rd }} 2005$. The questionnaire had been developed based on a series of interviews of active developers of XBox modifications including the webmasters of the online communities whose members we surveyed. ${ }^{11}$ The first community we targeted was Www.XBox-scene.com. According to its website,

"XBox-scene's primary goal is to keep it's visitors up-to-date about the XBox Scene. Unlike other sites, software pre/reviews aren't [...] major sections. XBox-Scene is specialized in hardware news and information. [XBox-scene] will talk about mods (modifications) and other hardware aspects." 12

The second online community we surveyed was the www.XBox-linux.org. While $\mathrm{XBox}-$ scene.com is a general interest community with an emphasis on the whole spectrum of possible modifications of the XBox, XBox-linux.org is closely related to the XBox Linux Project which

"[... aims to provide a version of GNU/Linux for the XBox, so that it can be used as an ordinary computer. Linux should make use of all XBox

\footnotetext{
${ }^{11}$ We conducted a total of 6 unstructured interviews of about 90 minutes length each prior to designing the questionnaire. Our interview partners include the administrators of $w w w . x b o x-l i n u x$. org, WWw.xbox-scene.com the designer of the SmarttXX modchip as well as three users owning a modified XBox.

${ }^{12}$ See http://www.XBox-scene.com/about.php for more details, latest visit on February, $26^{\text {th }}$, 2008.
} 
hardware and allow the user to install and run software from standard i386 Linux distributions". ${ }^{13}$

Our survey was advertised prominently on both web pages and resulted in a total of 2,256 responses. As data on the size of the basic population was not available we are unable to report a precise response rate. However, we conducted a non-response analysis comparing early to late respondents (Armstrong \& Overton 1977) which yielded no indication of a non-response bias. ${ }^{14}$

In our study, outlaw community members are defined as members who have modified their XBox to circumvent the XBox's security mechanism so as to be able to execute software which is not authorised by Microsoft (homebrew software and illegal copies of authorised software). Based on findings of Mollick (2004) that outlaw communities often consist of innovators and adopters, we aim to identify both groups of participants. We therefore asked our respondents to indicate whether they have actively contributed to the development of any unauthorised software code for the XBox. In total, $19.99 \%$ of the respondents are user innovators who contributed to unauthorised code while $80.01 \%$ did not contribute any modifications to the community and can be classified as adopters accordingly.

All respondents to our survey are male and between 14 and 61 years old. The average age of our respondents is 23 years. Although most of the respondents have a college degree or higher $(43 \%)$, about a fifth of them are still in school and approximately a third of them have only a high school degree. The majority of the respondents came from North America (U.S. 47.9\% of all respondents and Canada 9.7\% of all respondents). The remaining respondents are distributed more or less equally across Europe and the Asian-Pacific region.

\section{Empirical Results}

In the following two subsections, we present results of our survey which describe firstly the process of modifying the XBox and secondly the motivation to do so for all respondents of our survey. If appropriate, we will contrast the answers of user innovators and adopters in the surveyed communities in order to highlight relevant differences. In the last subsection of this section we present evidence of the major motivations of user innovators and compare them to previous evidence of user innovators which have been derived from surveys conducted within the open source software scene.

\footnotetext{
${ }^{13}$ See http: //www. XBox-linux.org/wiki/XBox-Linux: About, latest visit on February, $26{ }^{\text {th }}, 2008$.

${ }^{14}$ In particular, we compared the first $10 \%$ with the last $10 \%$ of the responses to our survey with regard to the means of relevant variables using T-tests. The observed differences are not significant and does not point to systematic differences between early and late responses.
} 
Table 1: Tabulation of how the modification of respondents' XBoxes was applied.

\begin{tabular}{|c|c|c|c|}
\hline $\begin{array}{l}\text { Who performed the modification } \\
\text { of your XBox? }\end{array}$ & Adopter & Innovator & Total \\
\hline I bought a modified XBox. & $\begin{array}{c}87 \\
(4.85 \%)\end{array}$ & $\begin{array}{c}12 \\
(2.68 \%)\end{array}$ & $\begin{array}{c}99 \\
(4.42 \%)\end{array}$ \\
\hline I did it on my own (without any help). & $\begin{array}{c}1,224 \\
(68.23 \%)\end{array}$ & $\begin{array}{c}351 \\
(78.52 \%)\end{array}$ & $\begin{array}{c}1,575 \\
(70.28 \%)\end{array}$ \\
\hline I did it with help from my friends. & $\begin{array}{c}262 \\
(14.60 \%)\end{array}$ & $\begin{array}{c}46 \\
(10.29 \%)\end{array}$ & $\begin{array}{c}308 \\
(13.74 \%)\end{array}$ \\
\hline $\begin{array}{l}\text { I shipped the XBox to a commercial } \\
\text { vendor who did the modification. }\end{array}$ & $\begin{array}{c}88 \\
(4.91 \%)\end{array}$ & $\begin{array}{c}15 \\
(3.36 \%)\end{array}$ & $\begin{array}{c}103 \\
(4.60 \%)\end{array}$ \\
\hline $\begin{array}{l}\text { People from the XBox } \\
\text { Community helped me. }\end{array}$ & $\begin{array}{c}55 \\
(3.07 \%)\end{array}$ & $\begin{array}{c}6 \\
(1.34 \%)\end{array}$ & $\begin{array}{c}95 \\
(4.24 \%)\end{array}$ \\
\hline Other & $\begin{array}{c}78 \\
(4.35 \%)\end{array}$ & $\begin{array}{c}17 \\
(3.80 \%)\end{array}$ & $\begin{array}{c}61 \\
(2.72 \%)\end{array}$ \\
\hline Total & $\begin{array}{c}1,794 \\
(100.00 \%)\end{array}$ & $\begin{array}{c}447 \\
(100.00 \%)\end{array}$ & $\begin{array}{c}2,241 \\
(100.00 \%)\end{array}$ \\
\hline
\end{tabular}

Note that 15 respondents did not answer the question.

\subsection{Modification and use of the XBox}

The disablement of the XBox's security mechanism is a prerequisite to the execution of non-authorised software code. There are various ways to circumvent the security mechanism but all of them require some modifications of the gaming console - either by modification of the hardware configuration or by modification of the embedded firmware. One way of performing the necessary modification is to open up the XBox and to solder in a modchip which is a specifically customised chip disabling the security mechanism. ${ }^{15}$ Alternatively, it is also possible to overwrite (flash) the XBox's ROMChip containing the embedded firmware with an alternative software (hardware hack). A third way is to exploit a buffer overrun by applying special software tools (software hack). In the latter two cases, it is not necessary to open up the XBox. According to our survey, approximately three quarters of the respondents $(78.6 \%)$ had soldered a modchip into their XBox, $23 \%$ had overwritten the ROM-Chip and $25 \%$ performed a software hack - multiple hacks at the same time are possible.

Table 1 presents results of how individuals modified their XBox: $70.3 \%$ of the respondents indicated that they had modified their XBox themselves while $13.7 \%$ were assisted by friends. Results differ slightly across innovators and adopters. A larger percentage of user innovators $(78.7 \%)$ modified their XBox themselves as compared

\footnotetext{
${ }^{15}$ Modchips for video gaming consoles are legally sold by third party manufacturers.
} 
Figure 1: Share of respondents who applied the following modifications to their XBox.

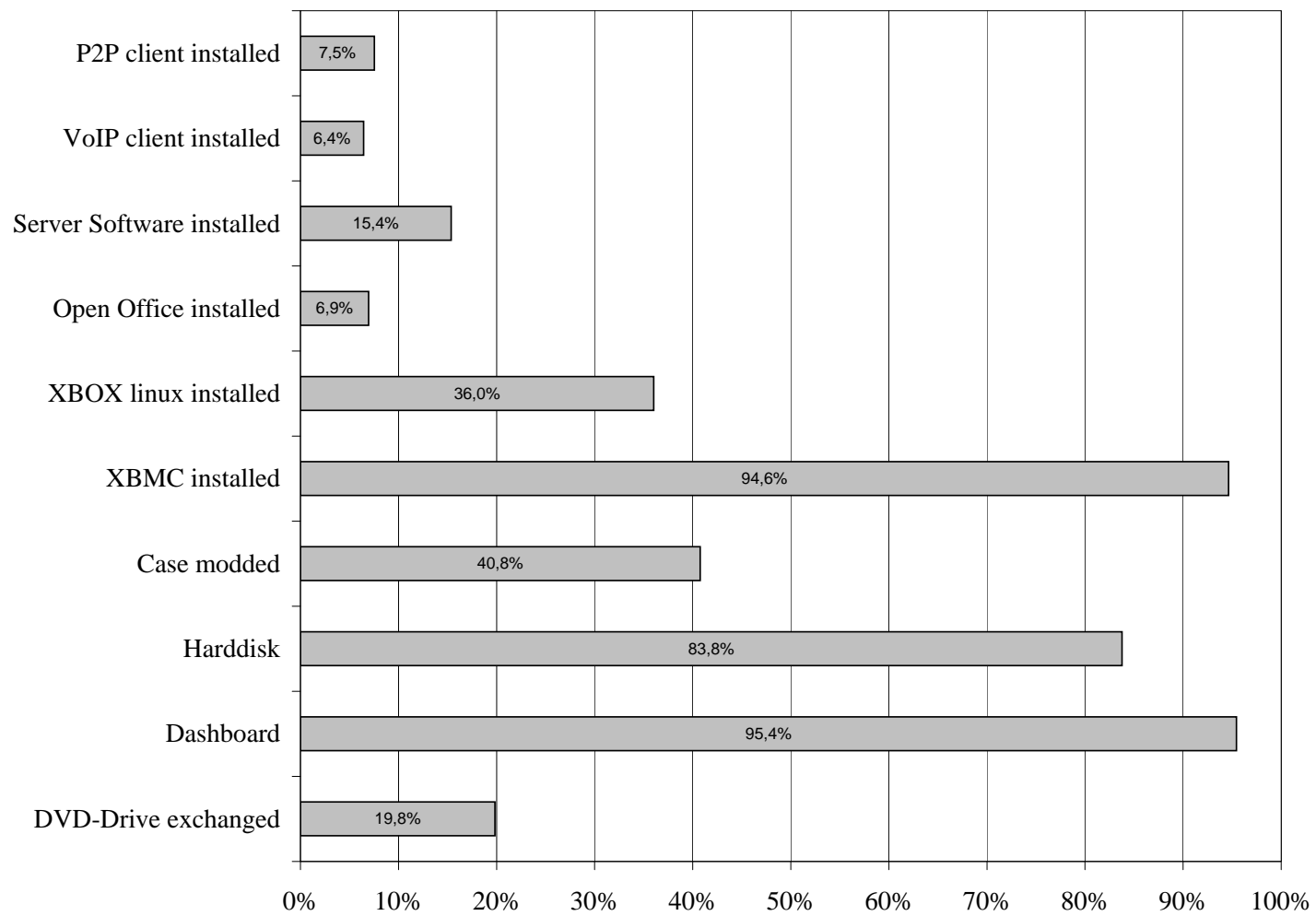

to the adopters $(68.2 \%)$. In a T-test we were able to reject the hypothesis that this difference is equal to zero at the $1 \%$ significance level.

Once the XBox has been modified in a way that the security mechanism is disabled different types of non-authorised software codes can be executed. Figure 1 shows the percentage of respondents who installed various types of software applications. In addition to the extension of the XBox's spectrum of functions by the installation of software, hardware modifications can be performed to improve the technical specifications of the console. For example, the XBox contains a standard IDE hard drive controller making it easy for users to exchange the original 10 GB hard disk by a larger standard 3.5" hard disk in order to increase storage capacity. Figure 1 shows that almost all participants had installed the XBox Media Center XBMC (94.6\% of all respondents) and amended the operating system (also called dashboard, 95.4\% of all respondents). In combination with the large fraction of respondents that exchanged the original hard disk $(83.8 \%)$, it can be assumed that most of the respondents are using the XBox as a multimedia center which enables the user to be able to store, manage and play different types of media files. This inference is supported from our interview evidence with active members of XBox user communities suggesting that the XBox with its direct TV-output is an ideal TV set-top box as it is not only capable to play from but also to rip DVDs and CDs directly to its harddisk. Note that the installation of software applications familiar from PCs (Linux, office applications, voice-over-IP-clients or also 
Figure 2: Frequency of alternative ways to use the XBox. Note: The first bar of each category corresponds to the answers provided by adopters. The second bar corresponds to the answers provided by user innovators.

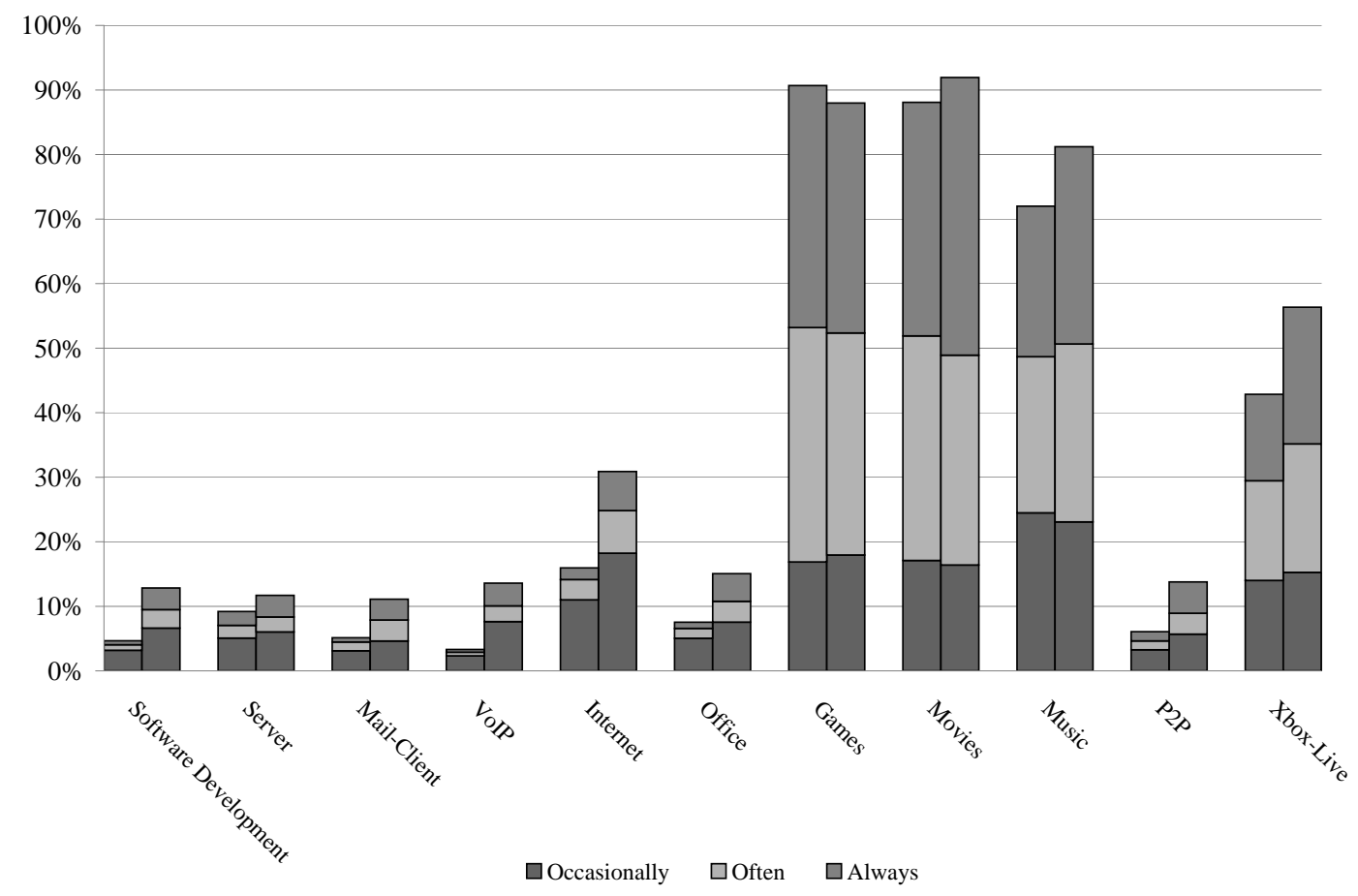

peer-to-peer-clients) do exist but are far less often installed than the ubiquitous XBMC.

We further investigated the usage of modified XBoxes as we asked users to indicate how often they use their XBox for certain purposes on a five-item scale (never, rarely, occasionally, often, always). Figure 2 presents the distribution of the answers separately for innovators and adopters. It can easily be seen that the large majority of the participants use the XBox primarily to play games, watch movies and listen to music. This result corresponds to the earlier finding that almost all participants had installed the XBox Multimedia Center which turns the XBox into a multi-media device enabling users to watch movies and listen to music - functions which are provided by Microsoft's original firmware only to a very limited extent. Interestingly, user innovators tend to use their XBox as a regular computer more often than adopters. They use the modified console more frequently for software development, as an internet-server or as mailclient, for telecommunication purposes (VOIP), to surf the internet, to run Microsoft Office applications and for file sharing over the internet (P2P).

\subsection{Motivation to modify the XBox}

In subsection 4.1 we described the process of modifying and the use of the modified XBox without taking reference to the motivation of the users to engage in this behavior. 
Figure 3: Importance of different motivational aspects to modify the XBox. Note: The upper bar of each category corresponds to the answers provided by innovators. The lower bar of each category corresponds to the answers provided by adopters. The neutral category "neither important nor unimportant" is not reported in the Figure.

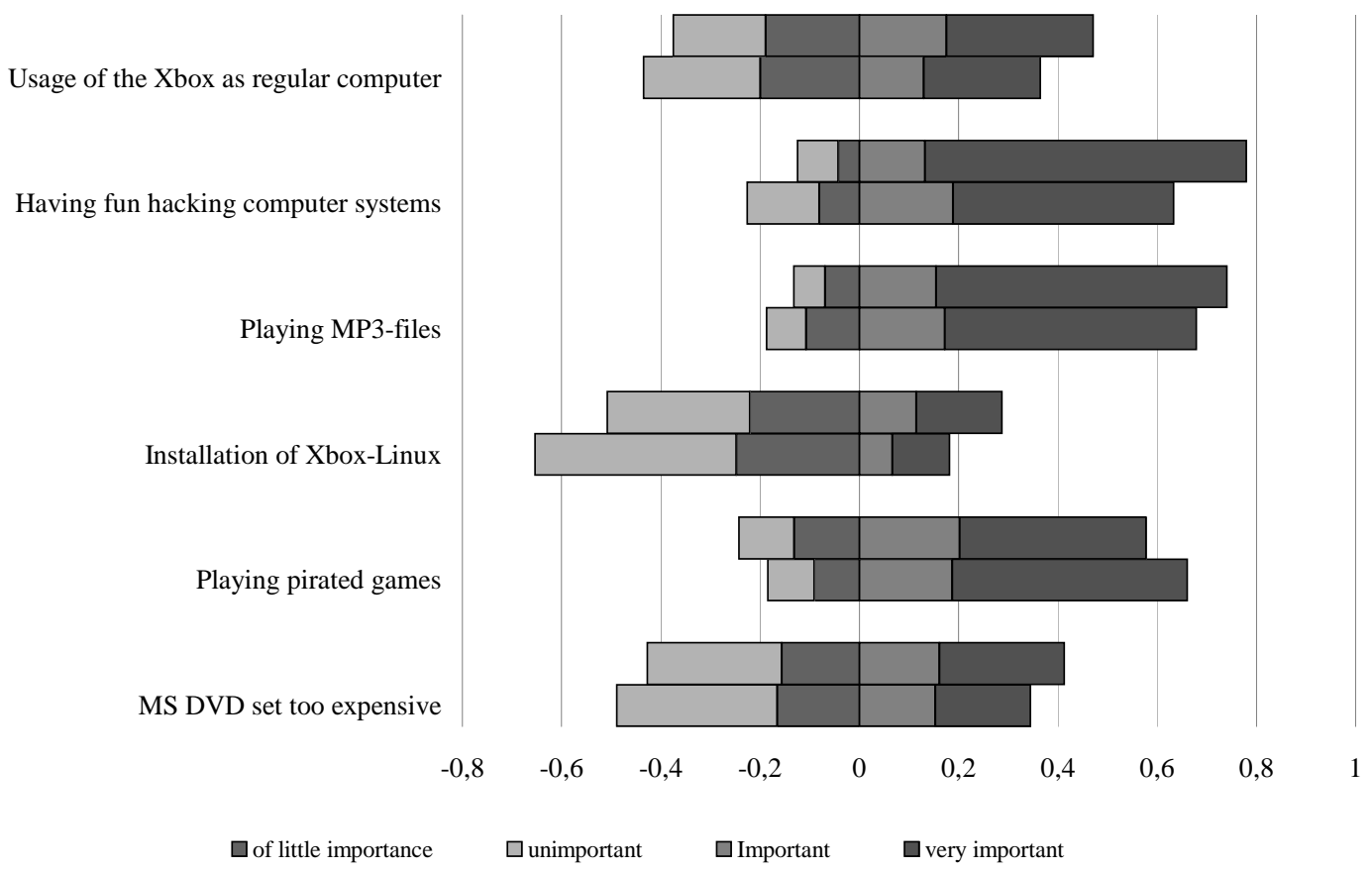

Motivational aspects deserve special attention here as the modification of the XBox often involves the breach of intellectual property rights ${ }^{16}$ and can therefore be regarded as an "outlaw" activity.

As indicated in Figure 3, one of the main motivations for users to modify their $\mathrm{XBox}$ is for the fun of hacking computer systems. Specifically, $63.4 \%$ of the adopters and $78.1 \%$ of the innovators indicated that this is an important to very important reason for modifying their XBox. We were able to reject the hypothesis that the difference between the two groups is equal to zero at the $1 \%$ level of significance. Another important motivation for hacking the console is to be able to execute illegal copies of games on the XBox. In particular, this aspect was rated as being important or very important by $66.1 \%$ of the adopters and $57.8 \%$ of user innovators. ${ }^{17}$ Results from statistical T-tests suggest that the difference between adopters and user innovators is different from zero at the $1 \%$ significance level. This latter finding support the findings of Mollick (2004), who found that user innovators tend to be more intrinsically

\footnotetext{
${ }^{16}$ Some of the tools enabling users to hack their XBox by modifying the embedded firmware violate Microsoft's IP rights.

${ }^{17}$ Additionally, we asked respondents to indicate how many games they owned in total and how many of those are original versions. On average, participants own approximately 53 games and only 13 are original.
} 
Figure 4: Agreement to different statements related to respondents attitude towards copyright protection of immaterial goods. Note: The upper bar of each category corresponds to the answers provided by innovators. The lower bar of each category corresponds to the answers provided by adopters. The neutral category "neither agree nor disagree" is not reported in the Figure.

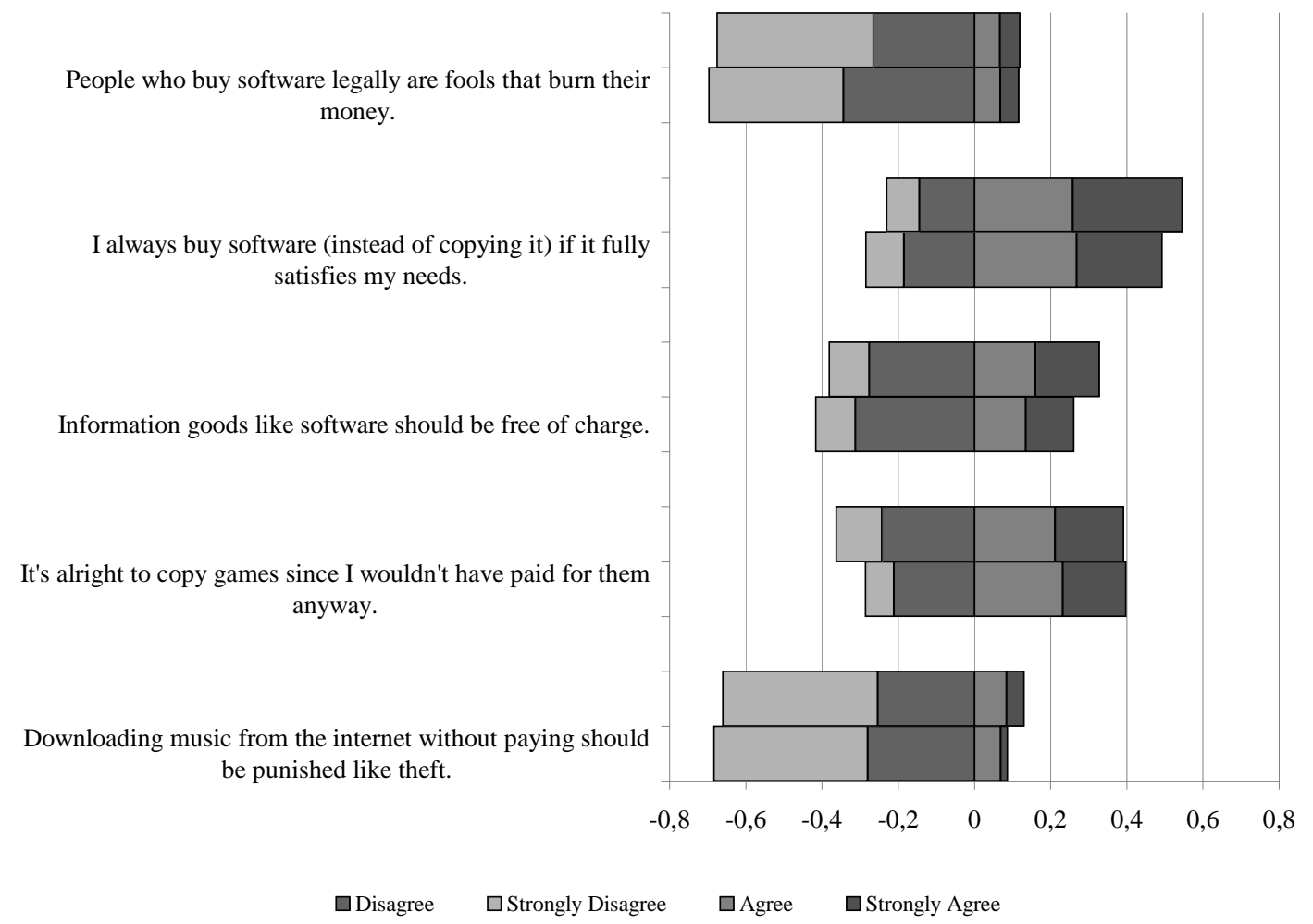

motivated to break into secured systems and argues that adopters tend to be vandals who are more motivated to adopt an outlaw innovation to gain unauthorised access to their product's system for deviant behaviour such as pirating authorised software.

Clearly, the ability to run illegal copies of games sold for the XBox is an important driver for users to modify their XBox. As we find differences across innovators and adopters we further investigated to which extent innovators and adopters also differ with respect to their attitudes towards deviant behaviour. Participants of our survey were asked to answer five general questions regarding their opinion and attitude towards copyright protection of immaterial goods like software or music in general. We included these questions to identify whether motivational differences between adopters and innovators are driven by their general attitudes or their evolvement in the development of homebrew software. Figure 4 presents differences in attitudes between adopters and innovators. On the aggregate level, it can be seen that participants are not all that deviant, as the majority feel that legally attained software is not a waste of money, that they would always pay for software if it fully satisfied their needs, and that information goods like software should not be made free of charge. Contrary to these findings, a large majority feel that it is alright to download music from the inter- 
Figure 5: Share of user innovators involved in the development of different types of applications.

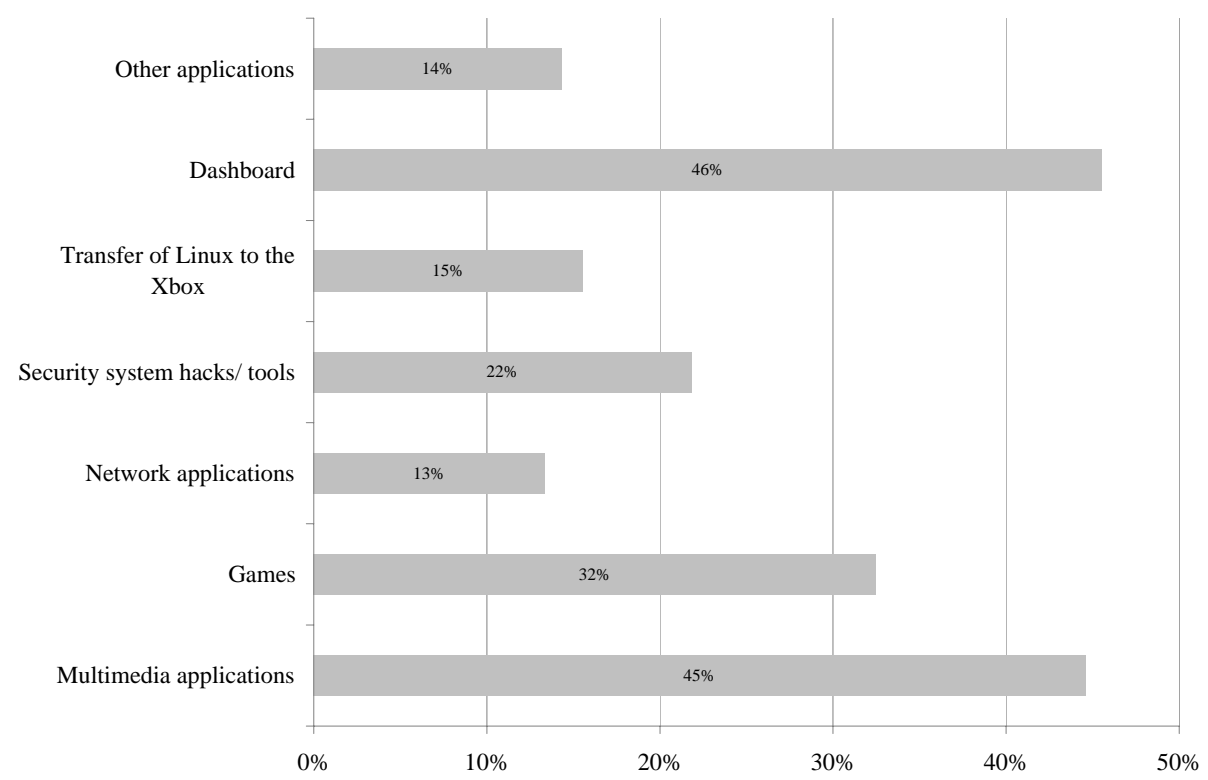

net without paying. It is worth noting that user innovators do not necessarily have a greater tendency to respect copyrights - in fact the differences of the distribution of the answers within the categories are not significantly different at the $5 \%$ level according to a $\chi^{2}$-test. We interpret this finding as an indication that the observed lower importance of the possibility to run pirated software among innovators compared to adopters (see Figure 3 is not driven by innovators' attitude towards copyrights in general.

\subsection{Characteristics of user innovators}

So far we reported results from our survey for all respondents distinguishing between innovators and adopters. As we are particularly interested in the motivation of user innovators to develop "outlaw" innovations we now turn to their characteristics. All statistics reported in the following refer exclusively to the responses given by the 447 innovators (about $19.99 \%$ of all respondents) in our sample.

With regard to the number of projects we observed that user innovators were involved in an average of 2.67 different projects when answering our survey. Figure 5 indicates the share of innovators involved in the different types of software applications. It can be observed that the development of alternative operating systems (dashboard, $46 \%$ ) and multimedia applications (46\%) are the most frequent categories for which user innovators develop software. This is followed by the development of add-ins for 
Figure 6: Motivation of user innovators to create software applications. Note: The neutral category "neither agree nor disagree" is not reported in the Figure. None of the respondents indicated "strongly disagree".

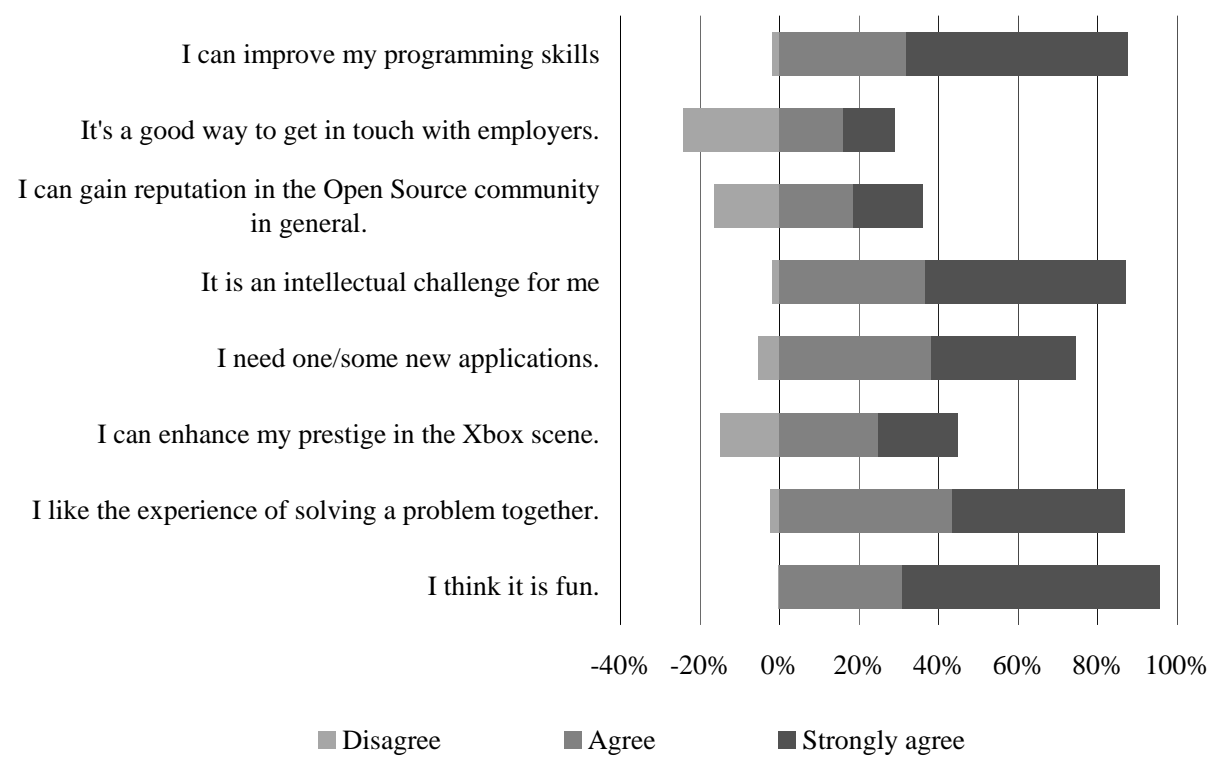

authorised games $(32 \%)$ and software hacks and tools to gain unauthorised access to the XBox's system (22\%). These findings are especially interesting as they provide evidence that users are not only just trying to gain unauthorised access to the XBox's system but that the majority of them are creating innovations to be able to use the system more effectively.

To better understand why user innovators are motivated to create applications, respondents were further asked to indicate the major reasons for their innovative activities. Figure 6 demonstrates that almost all user innovators $(96 \%)$ indicated that fun is a major incentive and $86 \%$ answered that they feel intellectually challenged when they create software applications for their XBox. Although these findings are much larger than those for open source software developers ${ }^{18}$, they support those of Mollick (2004) who found that user innovators tend to be intrinsically motivated by the desire to discover and innovate. Interestingly, we also find that a large majority of the innovators like the experience of solving problems together $(87 \%)$, suggesting that user innovators benefit by communicating with each other on innovation projects they are working on. This finding is consistent with what Shah $(\overline{2005 b})$ found that by working

\footnotetext{
${ }^{18}$ Lakhani \& Wolf 2005$)$ find that $20.3 \%$ of respondents in their sample indicated that they liked working with their particular open source development team and $44.9 \%$ indicated that writing code for the open source project was intellectually stimulating
} 
together with other users, user-innovators were able to access additional resources to develop and improve their innovations.

It can also be inferred from Figure 6 that extrinsic aspects are important for user motivation to innovate. In fact, $88 \%$ of the responding innovators indicate that the possibility to improve their programming skills motivates them to engage in the development of homebrew software. Further, innovators create applications because they need the applications for their own personal use. This result corresponds to the motivation proposed by von Hippel (1988) as to why users innovate. However, in contrast to findings of Mollick (2004), we find that majority of the user innovators are not motivated by reputation. Specifically, only $45 \%$ indicated that they innovate to enhance their prestige in the XBox scene and even less $(36 \%)$ indicated that they innovate to gain reputation in the open source software community in general.

\section{Conclusion}

It is a common phenomenon that users develop innovations which modify or improve products provided by a manufacturer. If these innovations extend the possible use beyond the use envisaged by the manufacturer they can be regarded as "outlaw" innovations in the sense of Flowers (2008). Our survey of participants of two online innovation communities built around outlaw innovations for Microsoft's XBox yielded interesting insights in the reasons why users engage in outlaw behaviour. Our findings are twofold: First, we found that most users modify/ hack their console in order to gain access to functionalities not provided by the manufacturer. In particular, almost all of the 2,256 users responding to our survey installed the XBox Media Center which provides much better multi-media functions than Microsoft's original firmware. Second, users are largely motivated to modify their XBox for the possibility to run pirated software but also for intrinsic motivations such as fun of hacking computer systems. With regard to the characteristics of user innovators, we find they are more intrinsically motivated than open source software developers. In particular, fun and intellectual stimulation are major reasons as to why they participate in the development of homebrew software.

Our findings suggest that the existence of outlaw communities favours the diffusion of outlaw innovation, such as that of the XBox Media Center which has been adopted by a large number of community members. In this respect, manufacturers benefit from the existence of such communities as they provide complements otherwise not available to their proprietary product, thus increasing its attractiveness. On the other hand, one other major finding is that participants in outlaw communities are also interested in pirate behaviour. In fact, we find that a large majority of games participants own are pirated. Outlaw communities provide them with necessary information and tools to engage in such pirate behaviour, which manufacturers perceive to be harmful to them. 
Therefore, future research should try to weigh the manufacturer's costs and benefits from the existence of outlaw communities. Our survey provides a first explorative starting point by attempting to clearly delineate what these costs and benefits from outlaw communities are. 


\section{References}

Armstrong, J. S. \& Overton, T. S. (1977), 'Estimating nonresponse bias in mail surveys', Journal of Marketing Research 14, 396-402.

Bitzer, J., Schrettl, W. \& Schröder, P. J. H. (2004), Intrinsic Motivation in Open Source Software Development, Nr. 2004/19, Diskussionsbeiträge des Fachbereichs Wissenschaft der Freien Universität Berlin.

Dahlander, L. \& Magnusson, M. G. (2005), 'Relationships between open source software companies and communities: Observations from Nordic firms', Research Policy 34(4), 481-493.

Flowers, S. (2006), Harnessing the Hackers: The Emergence and Exploitation of Outlaw Innovation, Cops working paper, University of Brighton.

Flowers, S. (2008), 'Harnessing the hackers: The emergence and exploitation of outlaw innovation', Research Policy 37(2), 177-193.

Franke, N. \& Shah, S. (2003), 'How Communities Support Innovative Activities: An Exploration of Assistance and Sharing Among End-Users', Research Policy 32(1), 157-178.

Ghosh, R. A., Glott, R., Krieger, B. \& Robles, G. (2002), Free/Libre and Open Source Software: Survey and Study, Technical report, International Institute of Infonomics, University of Maastricht and Berlecon Research GmbH.

Grand, J., Yarusso, A., Baer, R., Brown, M. \& Thornton, F. (2004), Game Console Hacking: Having Fun While Voiding Your Warranty, Syngress Publishing, Rockland, MA.

Hars, A. \& Ou, S. (2001), 'Working for free? motivations for participating in opensource projects', International Journal of Electronic Commerce 6(3), 25-39.

Hienerth, C. (2006), 'The commercialization of user innovations: the development of the rodeo kayak industry', R\&D Management 36(3), 273-294.

Jeppesen, L. B. \& Frederiksen, L. (2006), 'Why Do Users Contribute to Firm-Hosted User Communities? The Case of Computer-Controlled Music Instruments', Organization Science 17(1), 45-63.

Jeppesen, L. B. \& Molin, M. J. (2003), 'Consumers as Co-developers: Learning and Innovation Outside the Firm', Technology Analysis \& Strategic Management 15(3), 363-383. 
Lakhani, K. R. \& von Hippel, E. (2003), "How Open Source Software works: "Free" User-to-User Assistance', Research Policy 32(6), 923-943.

Lakhani, K. R. \& Wolf, R. G. (2005), Why Hackers Do What They Do: Understanding Motivation and Effort in Free/Open Source Software Projects, in J. Feller, B. Fitzgerald, S. Hissam \& K. R. Lakhani, eds, 'Perspectives on Free and Open Source Software', The MIT Press, Cambridge-London.

Lerner, J. \& Tirole, J. (2001), 'The Open Source Movement: Key Research Questions', European Economic Review 45(4-6), 819-826.

Lerner, J. \& Tirole, J. (2002), 'Some Simple Economics of Open Source', The Journal of Industrial Economics 50(2), 197-243.

Levy, S. (1984), Hackers: heroes of the computer revolution., Anchor Press/ Doubleday, New York.

Mayrhofer, P. (2005), Does Commercial Interest Hinder Innovation in User Communities? - A Theoretical and Empirical Study, unpublished Masters thesis, University of Munich.

Meyer, G. R. (1989), The Social Organization of the Computer Underground, unpublished masters thesis, Illinois: Northern Illinois University.

Mollick, E. (2004), Innovations from the Underground: Towards a Theory of Parasitic Innovation, unpublished masters thesis, Cambridge, MA: MIT Library.

Prügl, R. \& Schreier, M. (2006), 'Learning from leading-edge customers at The Sims: Opening up the innovation process using toolkits', R\&D Management 36(3), 237250.

Raymond, E. S. (1999), The Cathedral and the Bazaar, in 'The Cathedral and the Bazaar: Musings on Linux and Open Source by an Accidental Revolutionary', O’Reily \& Associates, Inc., Cambridge, pp. 27-78.

Ryan, R. M. \& Deci, E. L. (2000), 'Intrinsic and Extrinsic Motivations: Classic Definitions and New Directions', Contemporary Educational Psychology 25(1), 54-67.

Schulz, C. (2006), The Secret to Successful User Communities: An Empirical Analysis of Computer Associates' User Groups, Discussion Papers in Business Administration 2006-9, University of Munich, Munich School of Management.

Shah, S. (2005a), From Innovation to Firm \& Industry Formation: "Innovation Communities" in the Windsurfing, Skateboarding, and Snowboarding Industries, Working Paper 05-0107, University of Illinois Working Paper. 
Shah, S. K. (2005b), Open Beyond Software, in D. Cooper, C. DiBona \& M. Stone, eds, 'Open Sources 2.0: The Continuing Evolution', O'Reilly Media: Sebastopol, CA.

Soghoian, C. (2007), 'Caveat venditor: Technologically protected subsidized goods and the customers who hack them', Northwestern Journal of Technology and Intellectual Property 6, 46-72.

Takahashi, D. (2002), Opening the Xbox: Inside Microsoft's Plan to Unleash an Entertainment Revolution, Prima Publishing, Roseville, CA.

Torvalds, L. \& Diamond, D. (2001), Just for Fun: The Story of an Accidental Revolutionary, HarperBusiness, New York.

von Hippel, E. (1988), The Sources of Innovation, Oxford University Press, OxfordNew York.

von Hippel, E. (2005), Democratizing Innovation, The MIT Press, Cambridge-London.

von Hippel, E. (2007), 'Horizontal innovation networks - by and for users', Industrial and Corporate Change 16(2), 1--23.

Wellman, B., Boase, J. \& Chen, W. (2002), 'The networked nature of community on and off the internet', IT \& SOCIETY 1(1), 151-165.

Wenger, E. (2004), 'Knowledge Management as a doughnut: Shaping your knowledge strategy through communities of practice', Ivey Business Journal 68(3), 1-8.

Zeitlyn, D. (2003), 'Gift Economies in the Development of Open Source Software: Anthropological Reflections', Research Policy 32(7), 1287-1291. 